\title{
Optical Properties of PolyvinylPyrrolidone and Carboxymethyl Chitosan films
}

\author{
A.I. Aboud \\ Department of Physics \\ Faculty of Science, Cairo University \\ Giza, Egypt
}

\author{
K.H. Mahmoud \\ Department of Physics \\ Faculty of Science, Cairo University \\ Giza, Egypt
}

\begin{abstract}
Polyvinyl Pyrrolidone (PVP), Carboxymethyl Chitosan (CMCH) films and their 50/50(wt/wt $\%)(\mathrm{PVP} / \mathrm{CMCH})$ blend sample were prepared using casting technique. X-ray diffraction technique revealed that, the prepared samples were miscible. The optical absorption was recorded at room temperature in the wavelength range of 190-800 $\mathrm{nm}$. Fitting of absorbance data proved that the transition is indirect allowed with optical band gap, $\mathrm{E}_{\mathrm{opt}}$, of $3.60 \mathrm{eV}$ for pure PVP film and 3.94eVfor pure CMCH. The blend sample containing 50wt\% PVP has an optical band gapand band tail with 3.80and $0.40 \mathrm{eV}$.Variation of optical band gap and band tail values under blending of pure polymers gives an indication of structural modification of blends. Optical parameters such as refractive index, complex dielectric constant and color parameters were studied.
\end{abstract}

Keywords: PVP, CMCH, Optical parameters, Urbach energy, Band gap

\section{Introduction}

One of the most important techniques in polymer science is blending. It is an attractive, simple, and inexpensive technique for producing new polymeric material with tailored performance without completely synthesis of new materials [1].

Polyvinyl pyrrolidone (PVP) is an amorphous water soluble, inert, nontoxic, conjugated polymer. It has good environmental stability, easy process situation, excellent transparency, excellent biocompatibility and surface activity. It has several pharmaceutical applications [2] and has been used as lubricant and as a main component of temporary skin covers or wound dressings [3]. In addition, PVP has the ability for complex formation through hydrogen bond between its carbonyl group and hydroxyl containing polymers such as 
carboxymethyl chitosan and polyvinyl alcohol $[4,5]$.

Carboxymethyl chitosan $(\mathrm{CMCH})$ is watersoluble polysaccharide, has some unique properties such as high viscosity, large hydrodynamic volume, low toxicity, biocompatibility, antibacterial properties, antitumor activities and film and gel-forming capabilities, it is also has several applications,

\section{Experimental}

\subsection{Samples preparation}

PVP with average FW approximately 40000 was supplied by Fisher Scientific, Fisher Chemicals, USA. Carboxymethyl Chitosan with molecular weight $(249.1)_{n}$ was supplied by Santa Cruz biotechnology, Germany. The solution method was used to obtain film samples. Weighed amounts of PVP and $\mathrm{CMCH}$ were dissolved in double distilled water at room temperature using a magnetic stirrer. Solutions

\subsection{Spectroscopic measurements}

$\mathrm{X}$-ray diffraction analysis of the samples is carried out with a Bruker AXS D8 Discover diffractometer with GADDS (General Area Detector Diffraction System) operating with a $\mathrm{Cu}-\mathrm{K}_{\alpha}$ radiation source filtered with a graphite monochromator $(\lambda=1.5406 \AA)$. e.g., production of super absorbents, chelating agents, and bio-sorbents for wastewater treatment [6-8].CMCH films also are usually used as wound dressing (i.e, artificial skin),packaging films and coatings [6, 9].

The aim of the present work is to achieve blends with enhanced optical properties in order to use them in industrial applications such as, displays, electronic and optoelectronic devices.

of the two polymers were mixed together with different weight percentages to give the blend system xPVP-(100-x) CMCH; (x= 100, 50,0 wt $\%)$ and samples are designated as P100,P50 and P0 respectively. Films of suitable thickness ( $75 \mu \mathrm{m})$ were casted onto stainless steel Petri dishes, and then dried in an ovenat $50^{\circ} \mathrm{C}$ for about 24 hours until solvent was completely evaporated.

The absorption measurements were performed using Evolution E220 spectrophotometer over the range of $190-800 \mathrm{~nm}$. The color parameters and color difference data were calculated using the CIEL* $\mathrm{u}^{*} \mathrm{v}^{*}$ system

\section{Results and discussion}

\subsection{X-Ray diffraction profiles}


$\mathrm{X}$-ray diffraction (XRD) was performed to discover the amorphous and crystalline portions of PVP, CMCH and their blend sample. Fig.1 shows the typical XRD pattern of PVP, $\mathrm{CMCH}$ and P50 blend sample.

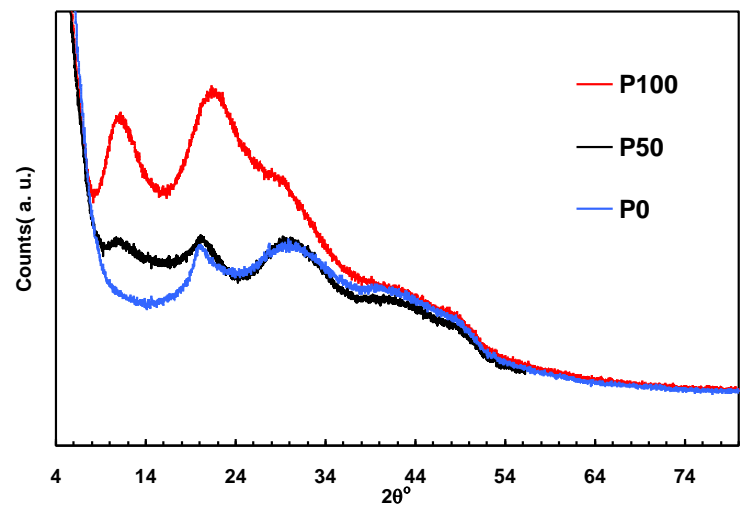

Fig.1 X-ray diffraction profiles of xPVP - (100-x) $\mathrm{CMCH}$ polymer blend system.

\subsection{Optical properties}

\subsubsection{Optical absorbance spectra}

Figure 2 shows the absorbance spectrum of xPVP - (100-x) CMCH polymer blend system from190-800 nm. The spectrum of Pure PVP (P100 sample) shows a shoulder like band at 206 $\mathrm{nm}$ and a band at $230 \mathrm{~nm}$.

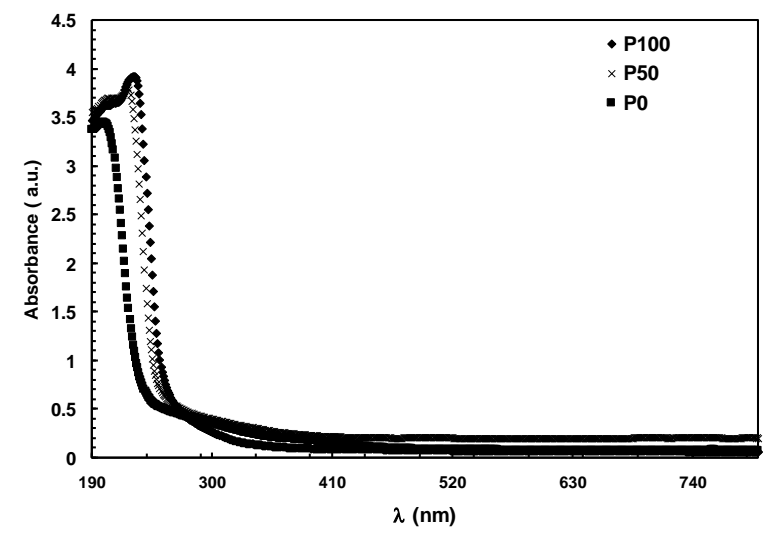

Fig.2 Absorbance spectra of xPVP - (100-x) CMCH polymer blend system

The observed shoulder and band may be attributed to $\pi \rightarrow \pi^{*}$ and $\mathrm{n} \rightarrow \pi^{*}$ electronic
The diffraction patterns showed that there is no sharp or narrow peak for $\mathrm{CMCH}$ and PVP indicating an amorphous nature. For the pure PVP (P100 sample), there are two peaks at around $2 \theta=11.3^{\circ}$ and $21.69^{\circ}$ with high intensity. The $\mathrm{CMCH}$ pure polymer (Po sample) exhibits two peaks at $20.24^{\circ}$ and $30.28^{\circ}$ and this is in a good agreement with literature [10]. For blend P50 blend sample, three peaks with small intensity appeared around $2 \theta=11.4^{\circ}, 20.59^{\circ}$ and $29.8^{\circ}$.From these observations one can conclude that, there is a certain level of miscibility between PVP and CMCH[11].

transitions respectively. In case of P50 sample the position of shoulder is approximately unchanged; on the other hand this shoulder located at $202 \mathrm{~nm}$ for $\mathrm{CMCH}$ sample (P0 sample).For P50 sample, a blue shift occurs for the band and this band disappears for P0 sample. It is observed that $\mathrm{CMCH}$ has lower absorbance values than other samples and PVP has the largest absorbance among employed samples in the wavelength range (190-300 $\mathrm{nm})$. The P50 sample has highest absorbance in visible region than other samples. The change in absorbance with composition is an indication of bonding between PVP and $\mathrm{CMCH}$ polymers .This bonding can occur through carbonyl group of PVP and hydroxyl group of $\mathrm{CMCH}[4,12]$.

\subsubsection{Optical parameters}

www.ijsea.com 
The absorption coefficient, $\alpha(v)$ below and near the absorption edge is related to absorbance through the relation:

$\alpha(v)=\frac{\log \left(\frac{1}{T}\right)}{t}$

Where $\mathrm{T}$ is transmittance and $\mathrm{t}$ is the film thickness.

The observed shift in the fundamental absorption edge of UV-visible spectral range can be related to the optical band gap by Tauc's expression $[13,14]$ :

$$
\alpha(v) E=C\left(E-E_{g}\right)^{q}
$$

where $\mathrm{C}$ is constant called band tailing parameter; $\mathrm{E}$ is the energy of incident photon $; \mathrm{E}_{\mathrm{g}}$ is the optical band gap energy and $q$ is the index such that $q=2,3,1 / 2,1 / 3$, that corresponds to indirect allowed, indirect forbidden, direct allowed and direct forbidden transitions, respectively.

Eq.2 as a function of wavelength $(\lambda)$ can be written as[15]:

Where $\lambda_{\mathrm{g}}$, is the wavelength corresponding to the optical band gap, and $\mathrm{c}$ is velocity of light. Using Eq.1, Eq.3 can be rewritten as:

$$
\frac{A(\lambda)}{\lambda}=S_{1}\left(\frac{1}{\lambda}-\frac{1}{\lambda_{g}}\right)^{q}+S_{2}(4)
$$

Where $S_{1}$ and $\mathrm{S}_{2}$ are constants. The parameter $\lambda_{\mathrm{g}}$ is obtained by extrapolating the linear portions of the $\left(\frac{A}{\lambda}\right)^{1 / q} v s 1 / \lambda$ curve at $\left(\frac{A}{\lambda}\right)^{1 / q}=0$
It was observed that the best fitting occurs for $\mathrm{q}=2$ and this corresponds to indirect allowed transition (Fig.3).

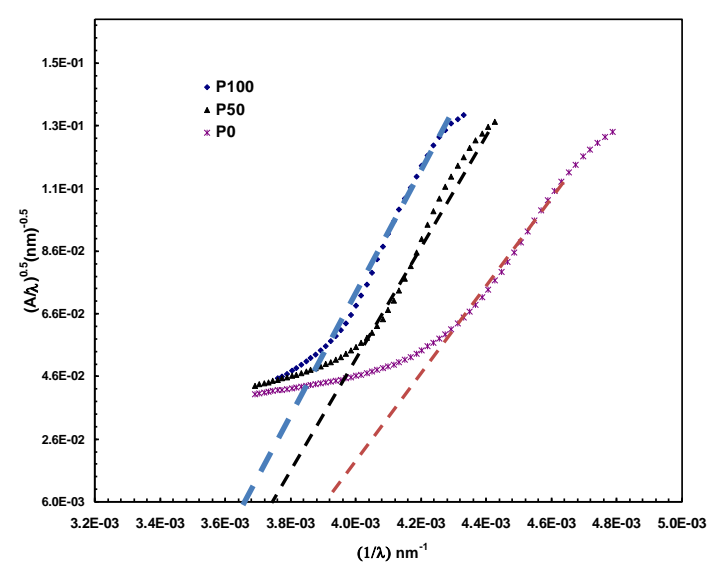

ASF plots of xPVP - (100-x) CMCH polymer blend system

It is observed that the optical energy gap values of blend sample is higher than that of PVP and lower than that of $\mathrm{CMCH}($ Table 1$)$.

The variation of band gap for blend is attributed to the structural and chemical bonds formation and this may suggest that there is a certain degree of miscibility in the blend due to the interaction between carbonyl groups or pyridine ring of PVP and hydroxyl groups of $\mathrm{CMCH}$

Table1. Values of optical band gap $\left(E_{\text {opt }}\right)$,Urbach energy $\left(E_{e}\right)$, high wavelength refractive index $\left(\mathrm{n}_{\mathrm{o}}\right)$ and number of carbon atoms $(\mathrm{M})$ per carbonaceous cluster for xPVP- (100-x) CMCH polymer blend system

polymers, respectively [16].Further, the values

\begin{tabular}{|c|c|c|c|c|}
\hline $\begin{array}{c}\text { Blend } \\
\text { number }\end{array}$ & $\mathrm{E}_{\text {opt }}(\mathrm{eV})$ & $\mathrm{E}_{\mathrm{e}}(\mathrm{eV})$ & $\mathrm{n}_{\mathrm{o}}$ & $\mathrm{M}$ \\
\hline P100 & 3.60 & 0.31 & 1.41 & $\sim 91$ \\
\hline P50 & 3.80 & 0.40 & 1.70 & $\sim 81$ \\
\hline P0 & 3.94 & 0.63 & 1.44 & $\sim 76$ \\
\hline & & & & \\
\hline
\end{tabular}

of optical band gap can be correlated to the number of carbon atoms per molecule through the expression [ 17] 
$M=\left(\frac{34.3}{E_{g}}\right)^{2}$

Where $\mathrm{M}$ is the number of carbon atoms in carbonaceous cluster. The values of $\mathrm{M}$ for PVP and $\mathrm{CMCH}$ are about 91, 76 respectively (Table 2). For blend sample the value of $M$ is between those of homopolymers and this can be correlated to the increased conjugation in monomer units of PVP and $\mathrm{CMCH}$ matrices after blending [18].

The band tail of localized states as stated by Urbach can be written as [19]

$$
\alpha(v)=F e^{\frac{h v}{E_{e}}}
$$

The width of the band tails $\left(E_{e}\right)$ associated with valence band and conduction bands was believed to be originated from the same physical origin [14]. In terms of absorbance Eq.6 can be written as:

$$
A(\lambda)=D e^{\frac{h c}{\lambda E_{e}}}
$$

Where $D$ is constant (=Ft/2.303). Obtaining the slope of linear regions of $\ln (A)$ vs. $1 / \lambda$ curves (Fig.4), the ( $\left.E_{\mathrm{e}}\right)$ values were calculated (Table 2).

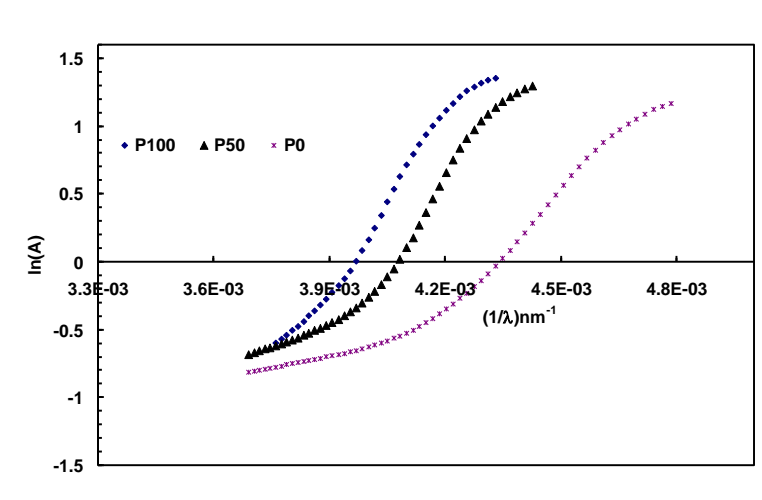

Fig.4 Plots of $\ln (\mathrm{A})$ vs. (1/ $\lambda)$ for xPVP - (100-x) $\mathrm{CMCH}$ polymer blend system

It is noted that the P0 sample has the largest tail width $\left(E_{e}=0.63 \mathrm{eV}\right)$, and P100 sample has the lowest value $(0.31 \mathrm{eV})$. For blend sample the value of tail width is between these values. The tail width gives an indication about disorder in samples and it's variation with composition could be attributed to miscibility between homopolymers. We also conclude that the blending process produces compounds with modified structural and optical properties.

\subsubsection{Refractive index dispersion}

The values of refractive index $\mathrm{n}$ and extinction coefficient $\mathrm{k}$ have been calculated using the theory of reflectivity of light $[20,21]$

$$
n=-\frac{(R+1)}{(R-1)} \pm 2 \sqrt{\frac{1-R}{1+k^{2}}+\left(\frac{R+1}{R-1}\right)^{2}}
$$

where $k=\alpha \lambda / 4 \pi$ is the extinction coefficient of the material. The dispersion of refractive for homopolymers and their blends is shown in Fig.5. 


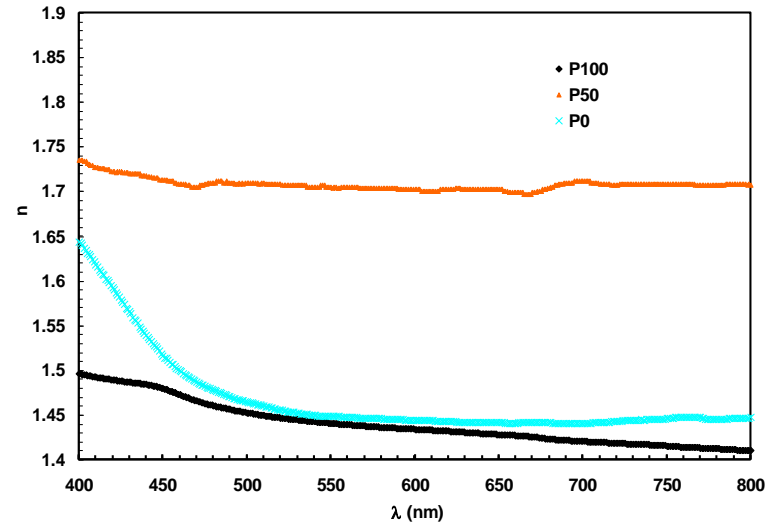

Fig.5 Variation of refractive index (n) with wavelength( ג) for xPVP - (100-x) CMCH polymer blend system It is clear that the P50 blend sample has largest refractive index among the employed samples in the wavelength range $539-800 \mathrm{~nm}$ and this can be attributed to the reduction in free volume in the polymer sample as a result of blending [22].

\subsubsection{Dielectric function}

The real and imaginary parts of the complex dielectric function are related to the refractive index through the following expressions [23]

$$
\varepsilon^{\prime}(\lambda)=n^{2}(\lambda)-k^{2}(\lambda)
$$

$$
\varepsilon^{\prime \prime}(\lambda)=2 n(\lambda) k(\lambda)
$$

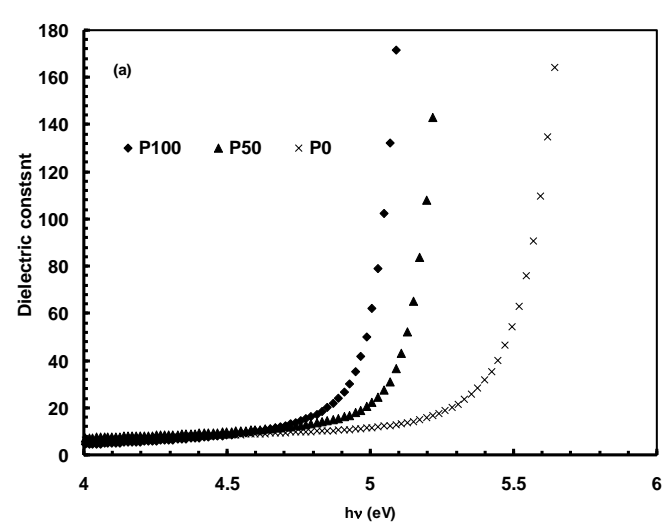

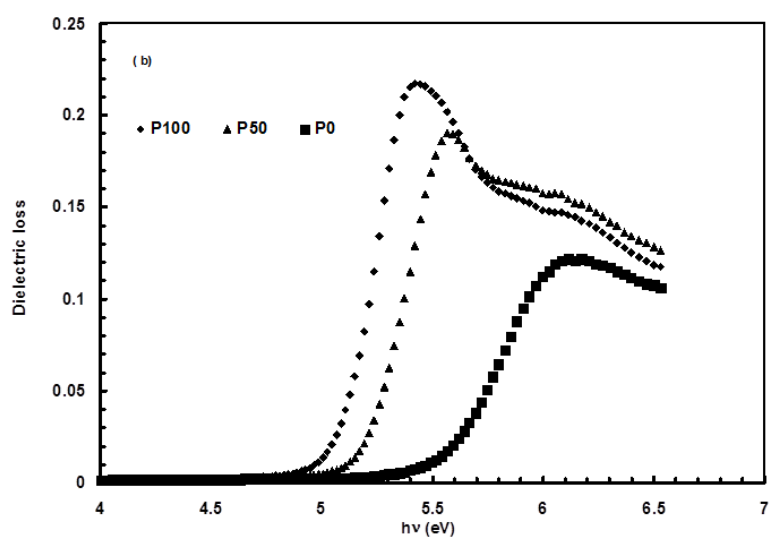

Fig.6 Plots of (a) dielectric constant, (b) dielectric loss versus photon energy (hv)for xPVP - (100-x) CMCH polymer blend system

A relaxation peak is observed in $\varepsilon^{\prime \prime}$ spectra at certain energies (larger than optical band gap values) as shown in (Fig. 6(a,b)).The dielectric constant and loss for P100 sample has larger values than other samples. The modification for pure polymers due to blending occurs via interaction through some functional groups such as carbonyl and hydroxyl groups on both polymers [16] and this can enhance the polarization processes in the samples and affect the dielectric parameters.

\subsubsection{Color study}

Tristimulus curves (Fig.7) show two peaks at about 560nm (higher intensity) and 470nm (lower intensity). Also it is observed that $\mathrm{Y}_{\mathrm{t}}(\max )$ varies with composition and $\mathrm{P} 100$ sample has higher $\left(\mathrm{Y}_{\mathrm{t}}\right)$ values over all wavelength range for all samples. 


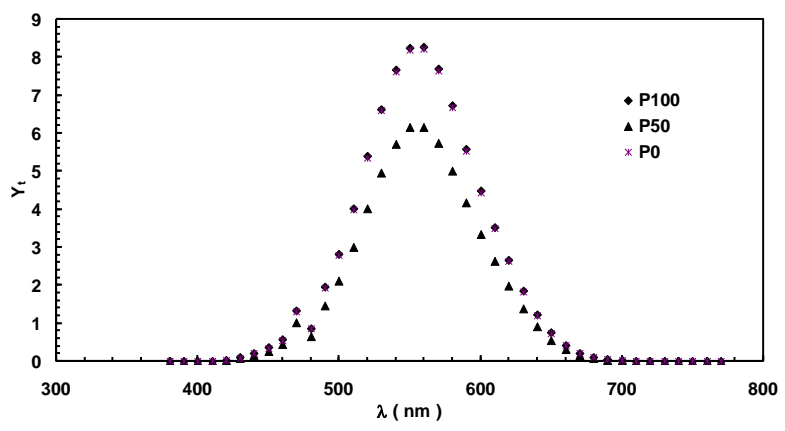

Fig.7 Tristimulus transmittance of xPVP - (100-x) $\mathrm{CMCH}$ polymer blend system

The color parameters $\mathrm{L}^{*}, \mathrm{U}^{*}, \mathrm{~V}^{*}, \mathrm{~h}_{\mathrm{ue}}, \mathrm{W}, \mathrm{Y}_{\mathrm{e}}$ [24] and color difference data, $\Delta \mathrm{L}^{*}, \Delta \mathrm{U}^{*}, \Delta \mathrm{V}^{*}$,

Table 2. Color parameters for xPVP- (100-x) CMCH polymer blend system

\begin{tabular}{|c|c|c|c|c|c|c|c|c|c|c|c|c|}
\hline $\begin{array}{c}\text { Blend } \\
\text { number }\end{array}$ & $\mathrm{L}^{*}$ & $\mathrm{U}^{*}$ & $\mathrm{~V}^{*}$ & $\mathrm{C}^{*}$ & $\Delta \mathrm{L}^{*}$ & $\Delta \mathrm{U}^{*}$ & $\Delta \mathrm{V}^{*}$ & $\Delta \mathrm{C}^{*}$ & $\Delta \mathrm{E}$ & $\mathrm{h}_{\mathrm{ue}}$ & $\mathrm{W}$ & $\mathrm{Y}_{\mathrm{e}}$ \\
\hline P100 & 93.37 & 0.66 & 2.78 & 2.86 & ----- & ----- & ----- & ---- & ---- & 76.62 & -1002.56 & 0.03 \\
\hline P50 & 83.19 & 0.37 & 0.94 & 1.00 & -10.18 & -0.29 & -1.84 & -1.86 & 10.34 & 68.79 & -747.88 & 0.01 \\
\hline P0 & 93.06 & 0.38 & 6.13 & 6.15 & -0.31 & -0.28 & 3.35 & 3.29 & 3.37 & 86.48 & -994.30 & 0.06 \\
\hline
\end{tabular}

$\Delta \mathrm{C}^{*}$, and $\Delta \mathrm{E}$ between blend samples and Pure PVP sample is shown in Table 2. It is observed that the P100 sample is lighter than all other samples. Also the P50 sample is greener, bluer and brighter than other samples. Also it has the highest total color difference value among all employed samples. The observed changes in the color parameters for polymer blend sample maybe due to the formation of new color centers.

\section{Conclusions}

PVP/CMCH blend system has been prepared by casting technique. XRD technique revealed that the prepared blends are miscible. Optical band gap, band tail width and refractive index are remarkably influenced by blending.The variation of optical and color parameters enables us to use these samples inelectronic and optoelectronic devices.

\section{References:}

[1]Peesan, M.,Supahol, P., \&Rujiravanit, R. 2005. Preparation and characterization of hexanoly chitosan/ polylactide blend films. Carbohydr.Polym., 60: 343-350

[2] Weber, H.A.,\&Molenaar, A.P. 1970. US patent 3557280

[3] Zheng, Y., Huang, X., Wang, Y., Xu, H., \&Chen, $\mathrm{X}$. 2009.Performance characterization of irradiated poly(vinyl alcohol)/polyvinyl pyrrolidone composite hydrogels used as cartilage replacement. J. Appl. Polym. Sci., 113:736-741

[4] Mosakala, E.J., Varnell, D.F., \&Coleman, M.1985. Concerning the miscibility of poly(vinyl phenol) blends - FTi.r. Study. Polymer, 26:228-234

[5] Goh, H.,\&Siow, K.S. 1990. Miscible blends of poly(N-vinyl pyrrolidone) with some hydroxyl-containing polymers. Polym. Bull., 23:205-209

[6] Lee, D.W., Hosun, L., Chong, H.N.,\& Shim, W.S. 2009. Advances in Chitosan Material and its Hybrid Derivatives: A Review. The Open Biomaterials Journal 1:10-20. 
[7]Zamani, A.,\&Taherzadeh, M.J.2010. Effects of partial dehydration and freezing temperature on water binding capacity of carboxymethyl chitosan-based superabsorbents. Ind. Eng. Chem. Res., 49: 8094-8099

[8]Tantala, J., Rachtanapun, C.,\&Rachtanapun, P. 2012.Effect of molecular sizes, sources of chitosan and plasticizer types on properties of carboxymethyl chitosan films. Adv. Mater.

Res., 506: 611-614

[9]Wang, L, Khor, E., Wee, A.,\& Lim, L.Y. 2002. Chitosan-alginate PEC membrane as a wound dressing: Assessment of incisional wound healing. J. Biomed.Mater. Res., 63:610618

[10]Tripathi, S., Mehrotra, G.K.,\& Dutta, P.K. 2009.Physicochemical and bioactivity of crosslinked chitosan-PVA film for food packaging applications. Int. J. Biol.Macromol., 45:372-37 [11] Reddy, K.S., Prabhakar, M.N., Reddy, V.N., Sathyamaiah, G., Maruthi, Y., Subha, M.C.S.,\& Rao, K.C. 2012. Miscibility studies of hydroxypropyl cellulose/poly(vinyl pyrrolidone) in dilute solutions and solid state.

J. Appl. Polym. Sci., 125:2289-2296

[12]Dayarian, S., Zamani,A., Moheb, A., \& Masoomi, M. 2014.Physico-Mechanical Properties of Films of Chitosan, Carboxymethyl Chitosan, and Their Blends. J. Polym. Environ., 22: 409 - 416

[13] Davis, E.A., \& Mott, N.F. 1970 Conduction in non-crystalline systems V.Conductivity, optical absorption and photoconductivity in amorphous semiconductors. Phil. Mag., 22: 903-922

[14]Tauc, J.,\&Menth, A.1972. States in the gap. J. Non-Cryst. Solids, 8: 569-585

[15]Ghobadi, N.2013.Band gap determination using absorption spectrum fitting procedure.Int.Nano.Lett., 3:1-4

[16]Liu, C., Xiao, C.,\&Liang, H. 2005. Properties and structure of PVP-lignin "blend films”. J. Appl. Polym.SCi., 95:1405-1411

[17]Fink, D., Chung, W.H.,Klett, R.,Schmoldt, A., Cardoso, J.,Montiel, R., Vazquez M.H., Wang, L., Hosoi, F., Omichi, H.,\& Langer, P.G. 2006. Carbonaceous clusters in irradiated polymers as revealed by UV-Vis spectrometry. Radiat.Eff.Def.Solids, 133:193-208

[18]Chahal, R.P., Mahendia, S.,Tomar, A.K.,\& Kumar, S.2011.Effect of ultraviolet irradiation on the optical and structural characteristics of in-situ prepared PVP-Ag nanocomposites. Digest. J. Nanomaterials\&biostructures,6:299306

[19]Urbach, F.1953.The Long-Wavelength Edge of Photographic Sensitivity and of the Electronic Absorption of Solids.Phys. Rev., 92:1324 
[20]Mahmoud, K.H. 2016.Optical properties

of hydroxyethyl cellulose film treated with

nitrogen plasma. SpectrochimActaA, 157: 153-

157

[21].Gosain, D.P., Shimizu, T., Ohmura, M.,

Suzuki, M., Bando, T., Okano, S.1991.Some

properties of $\mathrm{Sb}_{2} \mathrm{Te}_{3-\mathrm{x}} \mathrm{Se}_{\mathrm{x}}$ for nonvolatile memory based on phase transition. J .Mater. Sci., 26: 3271-3274

[22] Tyang, L.Y., Ramli, M.R., Othman, M.B.H., Ramli, R., Ishak, Z.A.M.,\& Ahmed ,Z.2013.Effect of crosslink density on the refractive index of a polysiloxane network based on $2,4,6,8$-tetramethyl-2, 4, 6, 8tetravinylcyclotetrasiloxane.Polym. Int. 62:382389

[23]Al-Ghamdi, A.A. 2006. Optical band gap and optical constants in amorphous $\mathrm{Se}_{96}$ ${ }_{x} \mathrm{Te}_{4} \mathrm{Ag}_{\mathrm{x}}$ thin films.Vacuum, 80:400405 [24]MacAdam, D.L., Color Measurement: Theme and Variations, Springer Series in

Optical Sciences (Springer - Verlag, BerlinHeidelberg -New York, 1981,vol.27) 